\title{
FINITELY GENERATED RESIDUALLY TORSION-FREE NILPOTENT GROUPS. I
}

\author{
GILBERT BAUMSLAG
}

\section{Dedicated to Mike (M. F.) Newman on the occasion of his 65th birthday}

(Received 29 January 1999; revised 9 June 1999)

\author{
Communicated by E. A. O'Brien
}

\begin{abstract}
The object of this paper is to study the sequence of torsion-free ranks of the quotients by the terms of the lower central series of a finitely generated group. This gives rise to the introduction into the study of finitely generated, residually torsion-free nilpotent groups of notions relating to the Gelfand-Kirillov dimension. These notions are explored here. The main result concerning the sequences alluded to is the proof that there are continuously many such sequences.
\end{abstract}

1991 Mathematics subject classification (Amer. Math. Soc.): primary 20F18.

Keywords and phrases: Residually torsion-free nilpotent groups, metabelian groups, algorithms.

\section{Introduction}

1.1. Basic objectives The objective of this paper is to transcribe into the study of the lower central series of a finitely generated group, ideas that have found much use in work on finitely generated associative algebras, see, for example, [14]. These ideas are the counterpart, in the case of lie algebras, to the notion of growth that has attracted a great deal of attention recently in group theory $[9,22]$.

Most of this work is devoted to finitely generated residually torsion-free nilpotent groups although the methods carry over also to the larger class of finitely generated residually nilpotent groups.

The author was supported in part by NSF Grant \#9626231.

(C) 1999 Australian Mathematical Society 0263-6115/99 \$A2.00+0.00 
In order to put this work into perspective, I have elected, in this rather leisurely introduction, to provide the reader with a little background before describing the new results obtained here.

1.2. Basic notation and definitions Let $G$ be a group and let $x_{1}, x_{2}, \ldots$ be elements of $G$. We denote the commutator $x_{1}^{-1} x_{2}^{-1} x_{1} x_{2}$ by $\left[x_{1}, x_{2}\right]$ and define, for $n>1$,

$$
\left[x_{1}, \ldots, x_{n+1}\right]=\left[\left[x_{1}, \ldots, x_{n}\right], x_{n+1}\right] \text {. }
$$

If $H$ and $K$ are subgroups of $G$, we define

$$
[H, K]=g p([h, k] \mid h \in H, k \in K) .
$$

The lower central series

$$
G=\gamma_{1}(G) \geq \gamma_{2}(G) \cdots
$$

of $G$ is defined inductively by setting

$$
\gamma_{n+1}(G)=\left[\gamma_{n}(G), G\right] .
$$

As usual, $G$ is nilpotent if $\gamma_{c+1}(G)=1$ for some $c$, with the least such $c$ the class of $G$.

Perhaps the most striking result in the theory of finitely generated nilpotent groups is the following theorem.

THEOREM 1.1. There is an algorithm which decides whether or not any two finitely generated nilpotent groups are isomorphic.

Theorem 1.1 is due to Grunewald and Segal (see [27]). It is a consequence of their work on algorithmic problems in rational arithmetic groups, which also contains a positive solution of the isomorphism problem for finitely generated nilpotent lie algebras over $\mathbb{Q}$, the field of rational numbers.

Theorem 1.1 allows one to think of finitely generated nilpotent groups as completely known.

Now the elements of finite order in a nilpotent group $H$ form a normal subgroup $\operatorname{tor}(H)$, the torsion subgroup of $H$. So it makes sense to define

$$
\bar{\gamma}_{n}(G) / \gamma_{n}(G)=\operatorname{tor}\left(G / \gamma_{n}(G)\right) .
$$

It is not too hard to deduce that

$$
\bar{\lambda}_{n}(G)=\bar{\gamma}_{n}(G) / \bar{\gamma}_{n+1}(G)
$$


is a torsion-free Abelian group. We define

$$
\bar{\gamma}_{\omega}(G)=\bigcap_{n=1}^{\infty} \bar{\gamma}_{n}(G)
$$

Then

DEFINITION 1. A group $G$ is termed residually torsion-free nilpotent if $\bar{\gamma}_{\omega}(G)=1$.

It follows that the residually torsion-free nilpotent groups consist of those groups $G$ such that for each $g \in G, g \neq 1$, there exists a normal subgroup $N$ of $G$, which may depend upon $g$, such that $g \notin N$ and $G / N$ is a torsion-free nilpotent group. Residually nilpotent groups can be defined in much the same way by simply relaxing the condition that $G / N$ be torsion-free nilpotent to nilpotent, without any extra qualification. Notice, in particular, that residually torsion-free nilpotent groups can be thought of as groups that can be approximated to by torsion-free nilpotent groups. It is on the class $\mathscr{T}$ of finitely generated residually torsion-free nilpotent groups that I want to concentrate here.

If $G=g p(S)$, then it turns out that

$$
\gamma_{n}(G) / \gamma_{n+1}(G)=g p\left(\left[x_{1}, \ldots, x_{n}\right] \gamma_{n+1}(G) \mid x_{i} \in S\right)
$$

This implies that every subgroup of a finitely generated nilpotent group is finitely generated. Consequently, if $G$ is finitely generated, then $\gamma_{n}(G) / \gamma_{n+1}(G)$ is a finitely generated Abelian group and $\bar{\lambda}_{n}(X)$ is a free Abelian group of finite rank, for every $n$.

1.3. Lower central Poincaré series and growth functions If $G$ is any finitely generated group, then as just noted, $\bar{\lambda}_{n}(G)$ is a free Abelian group of finite rank, say, $r(n)(=r(n, G))$. We consider the sequences

$$
r=r(G)=r(1), r(2), \ldots, r(n), \ldots
$$

and

$$
s=s(G)=s(1), s(2), \ldots, s(n), \ldots,
$$

where

$$
s(n)(=s(n, G))=\sum_{i=1}^{n} r(i)
$$


and we define $\delta(G)$ by

$$
\delta(G)=\limsup \frac{\log (s(n))}{\log n}
$$

Thus $\delta(G)$ is either a real number, or infinity, which reflects the rate of growth of the lower central series of $G$. We term $\delta(G)$ the lower central dimension of the group $G$. Let $\Phi$ be the class of all non-decreasing functions from the set of positive integers to the reals, which are ultimately positive-valued. If $f, g \in \Phi$ we define

$$
f \preceq g
$$

if there exists a positive integer $m$ such that

$$
f(n) \leq g(m n) \text { for all but finitely many } n .
$$

We define

$$
f \sim g \text { if } f \preceq g \text { and } g \preceq f
$$

This relation is an equivalence relation on $\Phi$ and we denote the equivalence class of $f \in \Phi$ by $\mathscr{F}$ and term $\mathscr{F}$ the growth off. We now define the lower central growth of the group $G$ to be the growth $\mathscr{S}$ of the function $s=s(G)$ defined above. One then says that lower central series of $G$ has polynomial growth if $\mathscr{S}=\mathscr{F}$, where $\mathscr{F}$ is the growth of a polynomial $f$. The corresponding notions of exponential growth and sub-exponential growth are defined in the analogous way. Notice that this notion of growth is different from that introduced by Milnor [22] involving word length.

My main concern is with the sequences $s$, the lower central dimensions and the lower central growths of finitely generated, residually torsion-free nilpotent groups, all of which are invariants reflecting some of the properties of the groups involved. Let $\rho(t)$ and $\sigma(t)$, where $t$ is here an indeterminate, be the corresponding generating functions:

$$
\rho(t)=\sum_{i=1}^{\infty} r(n) t^{n}, \quad \sigma(t)=\sum_{i=1}^{\infty} s(n) t^{n}
$$

Since

$$
\rho(t)=(1-t) \sigma(t)
$$

we lose nothing by restricting our attention to $\sigma(t)$, which we term here the lower central Poincaré series of the group $G$, which captures much of the nature of $s(G)$. 
1.4. Examples I want now to describe four families of residually torsion-free nilpotent groups. The first comes from free products where we have the following theorem.

THEOREM 1.2. The free product of residually torsion-free nilpotent groups is again residually torsion-free nilpotent.

This theorem is due to Malčev [19]. It follows, for example, that the free product of torsion-free nilpotent groups is residually torsion-free nilpotent. In particular free products of infinite cyclic groups, that is, free groups, are residually torsion-free nilpotent. This result was first proved by Magnus in 1935 [17]. In fact Magnus' basic idea plays a key role in the proof of Malčev's theorem.

It follows from the residual torsion-free nilpotence of free groups that the class of residually torsion-free nilpotent groups is not closed under quotients. It is however closed under subgroups and unrestricted direct products.

The next kind of example comes from the study of varieties of groups. Recall that a non-empty class $\mathscr{V}$ of groups is termed a variety if it is closed under subgroups, epimorphic images and unrestricted direct products. Typical of such a variety is the class $\mathscr{N}_{c}$ of all nilpotent groups of class at most $c$. Now given any pair of varieties $\mathscr{U}, \mathscr{V}$, one can form the so-called product variety $\mathscr{U} \mathscr{V}$ consisting of all extensions of groups in $\mathscr{U}$ by groups in $\mathscr{V}$. This product of varieties is associative. It turns the class of all varieties of groups into a free monoid (see [23]). Consider now the product variety

$$
\mathscr{V}=\mathscr{N}_{c_{1}} \cdots \mathscr{N}_{c_{k}}
$$

This variety $\mathcal{V}$, following Gruenberg [11] who invented it, is termed the polynilpotent variety of class row $\left(c_{1}, \ldots, c_{k}\right)$. In every variety there are the free groups (see, for example [24]). The point of these remarks is that Gruenberg [11], allying his work with that of Hall, proved in 1959 that

THEOREM 1.3. The free groups in the polynilpotent varieties are residually torsionfree nilpotent.

I come now to a family of examples, due to Lichtman [16]. Suppose that $L$ is a lie algebra over a commutative field $k$ of characteristic 0 . Then Cohn (see [8]) has proved that the universal enveloping algebra $U(L)$ of $L$ can be embedded in a field $F$. Lichtman proved the following theorem.

THEOREM 1.4. The multiplicative group $F^{\star}$ of $F$ is the direct product of the multiplicative group of $k$ and a group which is residually torsion-free nilpotent. 
It is worth noting that the universal enveloping algebra $U(L)$ can in general be embedded in fields that are essentially different from the one that Cohn manufactured. However if $L$ is nilpotent, then $U(L)$ is what is known as an Ore domain, that is, any two non-zero elements have a common, non-zero, right multiple. In this case $F$ is unique-indeed it coincides with the field of fractions of $U(L)$. Of course Lichtman's theorem applies to all such fields of fractions.

Notice that subgroups of $\mathscr{T}$-groups are residually torsion-free nilpotent. It follows that the fundamental groups of closed orientable surfaces belong to the class $\mathscr{T}$ by virtue of the following theorem [3].

THEOREM 1.5. Let $G$ be an amalgamated product of a finitely generated free group $F$ and a finitely generated free Abelian group A amalgamating a maximal cyclic subgroup of $F$ with a maximal cyclic subgroup of $A$. Then $G$ is residually free (and hence in $\mathscr{T}$ ).

It is clear from these remarks that the class of residually torsion-free nilpotent groups is a very rich one.

1.5. Recursive properties and problems I am now in a position to describe the new results that we prove in this paper, starting out with some algorithmic properties.

Notice first that finitely generated nilpotent groups have solvable word, conjugacy and isomorphism problems.

I mentioned the isomorphism problem at the outset. The positive solution of the word problem is probably due to Hirsch (see for example [15]), while the conjugacy problem was settled by Blackburn [7].

It follows from the positive solution of the word problem for finitely generated nilpotent groups and the fact that we can effectively compute the quotients $G / \gamma_{n}(G)$ of a finitely presented $\mathscr{T}$-group $G$, that finitely presented $\mathscr{T}$-groups have solvable word problem as well. It is not hard to also prove the following

THEOREM 1.6. The lower central Poincaré series of a finitely presented $\mathscr{T}$-group is recursive, that is, there is a general and effective procedure which computes the terms of the lower central Poincaré series of every $\mathscr{T}$-group.

There are, in addition to recursiveness, some other restrictions on the sequence $r=r(G)$ of a finitely presented $\mathscr{T}$-group $G$. For example

$$
r(n+1) \leq r(1) r(n) \leq r(1)^{n}
$$

and if $r(1)=1$, then $r(n)=0$ for every $n \geq 2$. If $r(n)=0$ for some $n$, then $r(m)=0$ for every $m>n$. In addition, if $F$ is a free group of rank $r(1, G)$, then it follows from 
the remarks after the formulation of Theorem 1.12 in Section 1.7, that

$$
r(n, G) \leq r(n, F), \text { for every } n .
$$

It appears to be a difficult problem to determine the precise nature of such sequences.

One can deduce, from the very formulation of Theorem 1.6, that the word problem for finitely presented $\mathscr{T}$-groups is, as noted earlier, solvable. Indeed the following theorem holds.

THEOREM 1.7. A recursively presented $\mathscr{T}$-group with recursive Poincaré series has a solvable word problem.

The proof of Theorem 1.7, although easy, is just a little more delicate than one might think at first.

As far as the conjugacy and isomorphism problems are concerned, the corresponding results for nilpotent groups do not carry over. Indeed, by modifying the groups involved in the fundamental work of Miller [20], we shall prove the following theorem.

THEOREM 1.8. There exists a finitely presented $\mathscr{T}$-group $G$ with an unsolvable conjugacy problem.

Since the conjugacy problem is solvable for finitely generated nilpotent groups, there exists a pair (indeed, infinitely many pairs) of elements of the group $G$ (in Theorem 1.8) which are conjugate modulo every term of the lower central series of $G$, but are not conjugate in $G$ itself.

Theorem 1.8 settles a question raised by Miller [21]. I believe that the isomorphism problem for finitely related $\mathscr{T}$-groups is also unsolvable, but the details of the proof that I have in mind, which is also based on Miller's work [20], are a little too involved to attempt inclusion at this time.

The recursive power series include the rational functions, that is, the quotients of two integral polynomials. It is to the rationality of lower central Poincare series of $\mathscr{T}$-groups that I want to turn next.

1.6. Rationality of lower central Poincaré series and dimension There is a naturally occurring class of $\mathscr{T}$-groups which have rational lower central Poincaré series.

THEOREM 1.9. Let the $\mathscr{T}$-group $G$ be Abelian-by-polycyclic. Then the lower central Poincaré series of $G$ is a rational function.

As usual a group $G$ is termed Abelian-by-polycyclic if it contains an Abelian normal subgroup $N$ such that $G / N$ is polycyclic. The polycyclic groups are the groups which 
can be built up from the the trivial group by repeatedly forming extensions by cyclic groups.

A sketch of the proof of Theorem 1.9 first appeared in [4] in 1990 in the case of Abelian-by-Abelian groups, that is, metabelian groups. This argument can be extended to Abelian-by-nilpotent groups and appears in print first in the paper of Groves and Wilson [10]. In fact the proof of Theorem 1.9 is not difficult. There are three steps. The first is to reduce the theorem to the case where $G$ is Abelian-bytorsion-free-nilpotent. The second is to notice, on appealing to a theorem of Jennings [13], that it suffices to prove an analogous result for graded modules over the group ring of a finitely generated torsion-free nilpotent group. The proof is then completed by mimicking an argument due to Hilbert and Serre about graded modules over finitely generated commutative algebras, as detailed, for example, in Atiyah and Macdonald [2]. The nilpotence of the group involved comes into play because of an induction which is fueled by using an element in the center. Since this part of the argument is contained in the cited work of Groves and Wilson, we will omit most of the details (see Section 3.2). In the case where $G$ is metabelian one can, with the aid of a theorem of Magnus (see [24, page 106]), deduce a little more.

COROLlaRY 1. Let the $\mathscr{T}$-group $G$ be metabelian. Then the following hold:

(1) For large enough values of $n, s(n)$ is a polynomial in $n$.

(2) $\delta(G)$ is a non-negative integer, namely the degree of the polynomial in 1 .

(3) If $G$ is a free metabelian group of finite rank $q>1$, then $\delta(G)=q$.

The ideas involved in the proof of Theorem 1.9 can be reworked to prove.

THEOREM 1.10. Let $G$ be a finitely generated Abelian-by-nilpotent group and let

$$
\Lambda(G)=\bigoplus_{n=1}^{\infty} \gamma_{n}(G) / \gamma_{n+1}(G)
$$

Then the following hold:

(1) The torsion subgroup of $\Lambda(G)$ is of finite exponent.

(2) For each prime $p$, if $e(n, p)$ is the order of the Sylow p-subgroup of the group $\gamma_{n}(G) / \gamma_{n+1}(G)$, then for large values of $n, e(n, p)$ is a polynomial in $n$.

(3) If, in addition, $G$ is polycyclic, then $\gamma_{n}(G) / \gamma_{n+1}(G)$ is ultimately of a fixed finite order.

Notice that it follows from Theorem 1.10 that only finitely many primes can arise as the orders of elements in the factor groups $\gamma_{n}(G) / \gamma_{n+1}(G)(n=1,2, \ldots)$, when $G$ is a finitely generated Abelian-by-nilpotent group. It is easy to see that this is not the case in general for finitely generated groups. Again there is here some overlap with the work of Groves and Wilson [10]. 
1.7. Lower central dimension and growth Hall in his work on finiteness conditions for soluble groups [12] has shown that there is a marked difference in the properties of finitely generated metabelian groups on the one hand, and finitely generated center-bymetabelian groups on the other. As far as the lower central dimension is concerned, this difference is not as marked. In fact finitely generated, center-by-metabelian groups, like finitely generated metabelian groups themselves, have finite lower central dimension. It turns out this property holds for an even more extensive class of groups.

THEOREM 1.11. The lower central dimension of a finitely generated, metanilpotent group, that is, nilpotent-by-nilpotent, is finite.

My original proof of the special case of Theorem 1.11 that deals with center-bymetabelian groups was rather elaborate. The referee was kind enough to point out that there is a simpler, more direct proof, which takes care of the case of nilpotentby-Abelian groups. I have made use of his idea to prove the even more general Theorem 1.11. I would like to thank the referee for the proof mentioned above and also for a number of helpful comments and a careful reading of this paper.

Notice that it follows from Corollary 1 (3) of Theorem 1.9 that every non-negative integer can occur as the dimension of some $\mathscr{T}$-group. I do not know what the possibilities are for the dimensions of $\mathscr{T}$-groups as a whole, indeed whether they are always integers. I will follow up on this question as well as carry out a general investigation of dimension, in particular of solvable groups, in the forthcoming paper [6].

It is not hard to deduce, either directly or from Witt's formula for the ranks of the successive quotients of the lower central series of a free group (see [18]), together with the remark in Section 1.5 following the formulation of Theorem 1.6, that

THEOREM 1.12. The lower central growth of a non-cyclic free group of finite rank is exponential and its lower central dimension is infinite.

Notice that if $G \in \mathscr{T}$, then $\delta(G)=0$ if and only if $G$ is nilpotent. It is easy to see that if $G \in \mathscr{T}$ and if $N$ is a normal subgroup of a finitely generated group $G$, then

$$
s(n, G / N) \leq s(n, G) \quad(n \geq 1), \quad \delta(G / N) \leq \delta(G) .
$$

One of the consequences of this remark, together with Theorem 1.12, is the following

COROLLARY 2. The lower central dimension of the free product of two non-trivial $\mathscr{T}$-groups is infinite.

It is of interest to remark here that Alperin and Peterson [1] have used Witt's formula to prove 
THEOREM 1.13. The lower central Poincaré series of a non-cyclic free group of finite rank, is not a rational function.

Indeed the irrationality of lower central Poincaré series is rather common, as we will see in Section 1.8, below.

1.8. Counting $\mathscr{T}$-groups The complexity of the class $\mathscr{T}$ is reflected by the following

THEOREM 1.14. Let $G$ be a $\mathscr{T}$-group whose center is not finitely generated and let $\mathscr{T}^{*}$ be the class of all quotient groups of $G$, whose centers are not finitely generated and which lie in $\mathscr{T}$. Then the set of lower central Poincare series of groups in $\mathscr{T}^{*}$ has the cardinality of the real line.

Hall [12] has constructed a 2-generator group $G$ which is center-by-metabelian and whose center is free Abelian of infinite rank. We shall show in Section 5.3 that $G \in \mathscr{T}$. It follows then from Theorem 1.14 that

COROLlARY 3. The lower central Poincaré series of 2-generator, center-by-metabelian groups in $\mathscr{T}$ can be put in a one-to-one correspondence with the set of all real numbers.

Thus there exist continuously many non-isomorphic 2-generator, center-by-metabelian $\mathscr{T}$-groups, with lower central Poincaré series which are not rational.

\section{Recursiveness}

2.1. Recursive lower central Poincaré series We begin with a sketch of the proof of Theorem 1.6, which should be compared with that of Theorem 1.7.

THEOREM 1.6. The lower central Poincaré series of a finitely presented $\mathscr{T}$-group is recursive.

PROOF. The left-normed commutators in a finite set of generators of $G$, of weight at most $n$, constitute, modulo $\gamma_{n+1}(G)$, a finite set of generators of $G / \gamma_{n+1}(G)$. These, together with a given finite set of defining relators of $G$ and the left-normed commutators of weight $n+1$ in the given generators of $G$, give rise to a finite presentation of $G / \gamma_{n+1}(G)$. We can thence effectively compute generators of the torsion subgroup of $G / \gamma_{n+1}(G)$. Consequently we can effectively compute finite presentations of the groups $\bar{\lambda}_{n}(G)$ and then, in turn, effectively compute their ranks. This proves Theorem 1.6. 
THEOREM 1.7. A finitely generated, recursively presented $\mathscr{T}$-group with a recursive Poincaré series, has a solvable word problem.

We begin the proof of Theorem 1.7 by first proving a few lemmas involving groups with recursive presentations.

LEMMA 1. Let $G$ be a group given by a finite set of generators and a recursively enumerable set of defining relations. Then $G / \gamma_{n}(G)$ has a presentation on the same set of generators together with a recursively enumerable set of defining relations.

PROOF. The proof of Lemma 1 is immediate. We simply add to the defining relators of $G$ all left-normed commutators of weight $n$ in all words in the given generators of $G$.

Our primary objective in regard to recording Lemma 1 is the following corollary to Lemma 1.

COROLLARY 4. Let $G$ be given by a finite set of generators linked by a recursively enumerable set of defining relations. Then $G / \bar{\gamma}_{n}(G)$ can be presented in the same way.

PROOF. The proof is straightforward enough. By Lemma 1 we can present $G / \gamma_{n}(G)$ on the given generators of $G$ in such a way that the set $R$ of all the relators involving these generators is recursively enumerable. Now the set $S$ of all $m$ th powers of all words in the generators of $G$, with $m$ here ranging over all positive integers, is recursively enumerable. It follows that

$$
T=\left\{w \mid w \in S, w^{m} \in R \text { for some } m>0\right\}
$$

is also recursively enumerable. But $T$ is a set of defining relators for $G / \bar{\gamma}_{n}(G)$. This proves the corollary.

We come now to the proof of Theorem 1.7 .

PROOF. We first show that we can effectively compute bases for the free Abelian groups $\bar{\lambda}_{n}(G)$. This will be accomplished inductively. Indeed we will concoct, for each positive integer $n$, a finite set $S_{n}$ of elements of $G$ that generate $\bar{\gamma}_{n}(G)$ modulo $\left[G, \bar{\gamma}_{n}(G)\right]$ and use $S_{n}$ to obtain a basis for $\bar{\gamma}_{n}(G)$ modulo $\bar{\gamma}_{n+1}(G)$. We begin by putting

$$
S_{1}=\left\{x_{1}, \ldots, x_{q}\right\}
$$

where here $\left\{x_{1}, \ldots, x_{q}\right\}$ is a finite set of generators of $G$. We proceed to obtain a basis for $\bar{\gamma}_{1}(G)=G$ modulo $\bar{\gamma}_{2}(G)$. We need to compare $r(1)$ with $q$. If $r(1)=q$, then 
modulo $\bar{\gamma}_{2}(G), x_{1}, \ldots, x_{q}$ is a basis for $\bar{\lambda}_{1}(G)$. If $r(1)<q$ then there is a dependence relation between $x_{1}, \ldots, x_{q}$ modulo $\bar{\gamma}_{2}(G)$. Thus as we recursively list the relators of $G$ we will come across a relation of the form

$$
x_{1}^{m_{1}} \cdots x_{q}^{m_{q}}=\text { a product of commutators, }
$$

where at least one of the $m_{i} \neq 0$. Now consider the Abelian group $A$ on $x_{1}, \ldots, x_{q}$ subject to the relation

$$
x_{1}^{m_{1}} \cdots x_{q}^{m_{q}}=1 \text {. }
$$

Then there is a Nielsen transformation $T$ of the absolutely free group on $x_{1}, \ldots, x_{q}$ such that $A$ can be presented as an Abelian group on the generators

$$
w_{1}(\vec{x})=T\left(x_{1}\right), \ldots, w_{q}(\vec{x})=T\left(x_{q}\right),
$$

(where the $w_{i}$ are written as non-Abelian group words in the original generators $x_{1}, \ldots, x_{q}$ ) subject to the relation

$$
w_{q}^{e_{q}}=1 \quad \text { in } A\left(e_{q}>0\right)
$$

Now if $r(1)=q-1$ it follows that $\left\{w_{1}, \ldots, w_{q-1}\right\}$ is a basis for $G$ modulo $\bar{\gamma}_{2}(G)$. If $r(1)<q-1$ then we can repeat the process applying it now to

$$
w_{1}(\vec{x}), \ldots, w_{q-1}(\vec{x})
$$

instead of to $x_{1}, \ldots, x_{q}$. If we now extend this Nielsen transformation to a Nielsen transformation of

$$
w_{1}(\vec{x}), \ldots, w_{q-1}(\vec{x}), w_{q}(\vec{x})
$$

which leaves $w_{q}$ fixed, then this process can be continued, ultimately yielding the following:

(i) a Nielsen transformation $U$ of the absolutely free group on $x_{1}, \ldots, x_{q}$ together with an integer $k$ such that if we set

$$
v_{1}=U\left(x_{1}\right), \ldots, v_{q}=U\left(x_{q}\right)
$$

then $v_{1}, \ldots, v_{k}$ constitute a basis for $G$ modulo $\bar{\gamma}_{2}(G)$;

(ii) each of $v_{k+1}, \ldots, v_{q}$ is of finite order modulo $\left[\bar{\gamma}_{1}(G), G\right]$.

It is important to note that we are not claiming that we can compute the orders of the elements $v_{k+1}, \ldots, v_{q}$ modulo $\left[\bar{\gamma}_{1}, G\right]$, but only that they are of finite order. We now put

$$
S_{2}=\left\{\left[v_{i}, v_{j}\right](1 \leq i, j \leq q), v_{k+1}, \ldots, v_{q}\right\}
$$


It follows that $S_{2}$ generates $\bar{\gamma}_{2}(G)$ modulo $\left[\bar{\gamma}_{2}(G), G\right]$, which completes the first step in the proof.

Now suppose that we have obtained a set $S_{n+1}$ which generates $\bar{\gamma}_{n+1}(G)$ modulo $\left[\bar{\gamma}_{n+1}(G), G\right]$. We now apply the same procedure to $S_{n+1}$ that we applied to $S_{1}$. The net result is that we end up with a Nielsen transformation of $S_{n+1}$ yielding a set $\left\{y_{1}, \ldots, y_{f}, y_{f+1}, \ldots, y_{h}\right\}$, which then generates $\bar{\gamma}_{n+1}(G)$ modulo $\left[\bar{\gamma}_{n+1}(G), G\right]$, such that

(i') $y_{1}, \ldots, y_{f}$ constitute a basis for $\bar{\gamma}_{n+1}(G)$ modulo $\bar{\gamma}_{n+2}(G)$;

(ii') the elements $y_{f+1}, \ldots, y_{h}$ are of finite order modulo $\left[\bar{\gamma}_{n+1}(G), G\right]$.

We are left with the choice of $S_{n+2}$. We work modulo $\left[\bar{\gamma}_{n+2}(G), G\right]$. This means that we may assume that $\bar{\gamma}_{n+2}(G)$ is central. It follows from (i') and $\left(i i^{\prime}\right)$ that $\bar{\gamma}_{n+2}(G)$ is generated by

$$
y_{f+1}, \ldots, y_{h} \text { together with }\left[\bar{\gamma}_{n+1}(G), G\right]
$$

Now

$$
\bar{\gamma}_{n+1}(G)=g p\left(S_{n+1},\left[\bar{\gamma}_{n+1}(G), G\right]\right) .
$$

Since $\bar{\gamma}_{n+2}(G)$ is central it follows from the usual commutator identities that

$$
\left[\bar{\gamma}_{n+1}(G), G\right]=g p\left(\left[y_{i}, x_{j}\right](i=1, \ldots, h, j=1, \ldots, q)\right) .
$$

Hence

$$
\bar{\gamma}_{n+2}(G)=g p\left(y_{f+1}, \ldots, y_{h},\left[y_{i}, x_{j}\right](i=1, \ldots, h, j=1, \ldots, q)\right) .
$$

We put

$$
S_{n+2}=\left\{y_{f+1}, \ldots, y_{h},\left[y_{i}, x_{j}\right](i=1, \ldots, h, j=1, \ldots, q)\right\} .
$$

This then completes the first part of the proof of Theorem 1.7.

Our final objective is to show how this solves the word problem for $G$. To this end let $w$ be any given word in the generators of $G$. Then we first begin to enumerate the (recursively enumerable) set of all relators of $G$ and check to see whether $w$ lies in this set. Second we enumerate the (recursively enumerable) set of all the relators of $G / \bar{\gamma}_{2}(G)$ and check to see if $w$ lies in this set. And thirdly we enumerate the (recursively enumerable) set of all words of the form $w w^{\prime}$ where $w^{\prime}$ ranges over all the words of the form $v_{1}^{e_{1}} \ldots v_{k}^{e_{k}}$, where not all the exponents $\boldsymbol{e}_{i}$ are zero (note $\left\{v_{1}, \ldots, v_{k}\right\}$ is a basis for $\bar{\gamma}_{1}(G)$ modulo $\left.\bar{\gamma}_{2}(G)\right)$ and check to see whether any of these words lies in the set of relators of $G / \bar{\gamma}_{2}(G)$. If the first eventuality holds, then $w=1$ in $G$; if the third one holds, then $w \neq 1$ in $G$; and finally if the second one holds, then $w \in \bar{\gamma}_{2}(G)$. If $w \in \bar{\gamma}_{2}(G)$ we repeat the process above replacing $\bar{\gamma}_{2}(G)$ by $\bar{\gamma}_{3}(G)$. Since $G \in \mathscr{T}$, at some stage we find either that $w=1$ in $G$ or that $w \neq 1$ in $G$, as desired. 
We noted in the introduction that the word problem is solvable for finitely presented $\mathscr{T}$-groups. By way of contrast, we demonstrate next that there exist finitely presented $\mathscr{T}$-groups with unsolvable conjugacy problems.

THEOREM 1.8. There exists a finitely presented $\mathscr{T}$-group with an unsolvable conjugacy problem.

The proof of Theorem 1.8 is based on that of an analogous theorem of Miller [20]. Indeed Theorem 1.8 is proved by a tiny modification of Miller's construction, on invoking the following.

LEMMA 2. Suppose that $G$ is a semi-direct product of $B$ and $Y$. If $B$ and $Y$ are residually torsion-free nilpotent and $Y$ acts trivially on $B / \bar{\gamma}_{2}(B)$, then $G$ is residually torsion-free nilpotent.

PROOF. It is enough to prove that if $x \in B, x \neq 1$, then we can find a normal subgroup $N$ of $G$ with torsion-free nilpotent quotient and $x \notin N$. Now there exists an integer $n$ such that $x \notin \bar{\gamma}_{n}(B)$. The group of those automorphisms of $B / \bar{\gamma}_{n}(B)$ that act trivially modulo $\bar{\gamma}_{2}(B) / \bar{\gamma}_{n}(B)$ is a torsion-free nilpotent group of class at most $n-1$. It follows that the canonical homomorphism of $Y$ into the group of automorphisms of $B / \bar{\gamma}_{n}(B)$ maps $Y$ onto a torsion-free nilpotent group of class at most $n-1$. If $K$ is the kernel of this homomorphism, then $K$ centralises $B$ modulo $\bar{\gamma}_{n}(B)$. Hence $\bar{\gamma}_{n}(B) K$ is a normal subgroup of $G, G / \bar{\gamma}_{n}(B) K$ is torsion-free nilpotent and $x \notin \bar{\gamma}_{n}(B) K$.

In order to complete the proof of Theorem 1.8, we consider

$$
U=\left\langle s_{1}, \ldots, s_{n} ; R_{1}, \ldots, R_{m}\right\rangle,
$$

a finitely presented, torsion-free group with an unsolvable word problem. So we may assume that the generators $s_{i}$ are all of infinite order. Let $F_{i}=\left\langle a_{i}, b_{i}\right\rangle$ be a free group of rank two $(i=1, \ldots, n)$. Then we successively form the amalgamated products

$$
U_{1}=\left\{U \star F_{1} ; s_{1}=\left[a_{1}, b_{1}\right]\right\}, \quad \ldots, \quad U_{n}=\left\{U_{n-1} \star F_{n} ; s_{n}=\left[a_{n}, b_{n}\right]\right\} .
$$

$U_{n}$ can be finitely presented on the generators $a_{1}, b_{1}, \ldots, a_{n}, b_{n}$ and defined in terms of these generators by finitely many relators all of which lie in the derived group of the free group on $a_{1}, b_{1}, \ldots, a_{n}, b_{n}$. Now Miller's argument [20] applied to $U_{n}$ yields a group $G$ with an unsolvable conjugacy problem. $G$ is actually a semi-direct product of the form prescribed by Lemma 2 . This proves Theorem 1.8.

I believe that a modification of the work of Miller [20, Theorem 26] together with Lemma 2, and an adaptation of an argument of Rabin [25], will yield a negative 
answer to the isomorphism problem for finitely related $\mathscr{T}$-groups. As I remarked in the introduction, clarification of the details of the argument that I have in mind will be left to another time.

\section{Rational functions and Abelian-by-polycyclic groups}

3.1. Lower central Poincaré series of Abelian-by-polycyclic groups Our objective in this subsection is to sketch the proof of Theorem 1.9 (Section 1.6).

THEOREM 1.9. Let the $\mathscr{T}$-group $X$ be-Abelian-by polycyclic. Then the lower central Poincaré series for $X$ is a rational function.

Theorem 1.9 is closely related to a similar theorem, Theorem 2.2 proved by Groves and Wilson in [10] (refer to Section 2 of that paper) and we refer to their work as needed here. A key step in the proof of Theorem 1.9 is the following proposition.

Proposition 1. Let $X$ be residually torsion-free nilpotent and let $A$ be a selfcentralising Abelian normal subgroup of $X$. Then $X / A$ is also residually torsion-free nilpotent

We shall need the following lemma.

LEMMA 3. Let $\Gamma$ be a subgroup of the group of automorphisms of the torsion-free Abelian group $A$. If $\Gamma$ acts nilpotently on $A$, then $\Gamma$ is torsion-free.

Lemma 3 is well-known and so we omit the proof - a proof can be easily fashioned after that of Lemma 2.

We come now to the proof of Proposition 1.

Proof. Suppose that $x \in X$ and that $x \notin A$. Then $[x, A] \neq 1$ since $A$ is selfcentralising. Consequently $[x, A] \notin \bar{\gamma}_{n}(X)$ for some $n$. So $x \bar{\gamma}_{n}(X)$ does not lie in the centraliser $C_{n} / \bar{\gamma}_{n}(X)$ of $A \bar{\gamma}_{n}(X) / \bar{\gamma}_{n}(X)$ in $X / \bar{\gamma}_{n}(X)$. By Lemma $3, X / C_{n}$ is torsion-free. Moreover $X / C_{n}$ is a quotient of $X / \bar{\gamma}_{n}(X)$ and so is nilpotent. This completes the proof of Proposition 1.

COROLLARY 5. If $X$ is residually torsion-free nilpotent, if $A$ is a self-centralising Abelian normal subgroup of $X$ and if $X / A$ is polycyclic, then $H=X / A$ is torsion-free nilpotent.

PrOOF. Since $H$ is polycyclic there is a bound on the torsion-free ranks of the quotients $H / \bar{\gamma}_{n}(H)$. Consequently the series $\bar{\gamma}_{n}(H)(n=1, \ldots)$ must stabilise. But, by Proposition $1, H$ is residually torsion-free nilpotent. Therefore $\bar{\gamma}_{n}(H)=1$ for a sufficiently large choice of $n$, that is, $H$ is nilpotent. 
Next we need to demonstrate the existence of an Abelian normal subgroup of $X$ which is self centralising.

LEMMA 4. Let the group $X \in \mathscr{T}$ and let $A$ be a maximal, Abelian normal subgroup of $X$ with nilpotent quotient $X / A=H$. Then $A$ is self-centralising and $H$ is torsionfree.

Proof. We prove, in the usual way, that $A$ is self-centralising. To this end let $C$ be the centraliser of $A$ in $X$. Since $C$ is a normal subgroup of $X$, if $C \neq A$ then $C / A$ is a non-trivial normal subgroup of the nilpotent group $H$. Hence $C / A$ meets the center of $H$ non-trivially. This means that we can find $x \in C, x \notin A$ with $x A$ in the center of $H$. Then $g p(x, A)$ is an Abelian normal subgroup of $X$ properly containing $A$, contradicting the maximality of $A$.

Next we prove that $H$ is torsion-free. Indeed, since $X$ is residually torsion-free nilpotent, $X$ is a group in which extraction of $n$th roots is unique, whenever they exist, for every positive integer $n$ (see for example [15, volume 2]). Now the centraliser $C$ of a subset in such a group where $n$th roots are unique is isolated, that is, if $a^{n} \in C$, for some $n>0$, then $a \in C$ (we refer to [15, volume 2] for details concerning the above and related notions). So $A$ is isolated and therefore $H$ is torsion-free.

3.2. The proof of Theorem 1.9 Let $G$ be a finitely generated, Abelian-by-polycyclic $\mathscr{T}$-group. By Lemma 4 and Corollary 5, we can find an Abelian normal subgroup $A$ of $G$ which is self-centralising and such that $H=G / A$ is torsion-free nilpotent. Let $R$ be the rational group algebra of $H$ and let

$$
M=A \otimes \mathbb{Q}
$$

Then $M$ can be turned into a right $R$-module in the usual way, via conjugation. Let $\mathscr{Q}$ be the augmentation ideal of $R$. Notice that there is an integer $n_{0}$ such that

$$
\gamma_{n_{0}}(G) \subseteq A
$$

Put $N=\gamma_{n_{0}}(G) \otimes \mathbb{Q}$. Then $N$ is an $R$-submodule of $M$. If $\operatorname{dim}(W)$ denotes the dimension of a rational vector space $W$, it is not hard to see that

$$
r(n)=\operatorname{dim}\left(N \mathscr{Q}^{n-n_{0}} / N \mathscr{Q}^{n-n_{0}+1}\right) \quad\left(n \geq n_{0}\right),
$$

where $r(n)$ is, as before, the rank of $\bar{\lambda}_{n}(G)$. We form now the graded algebra $\operatorname{Gr}(R)$ of $R$ using the powers $\mathscr{Q}^{n}$ of $\mathscr{Q}$ in the usual way (see for example [2]):

$$
\mathrm{Gr}(R)=\bigoplus_{n=0}^{\infty} \mathrm{Gr}_{n}(R)
$$


So

$$
\mathrm{Gr}_{0}(R)=\mathbb{Q} \quad \text { and } \quad \mathrm{Gr}_{n}(R)=\mathscr{Q}^{n} / \mathscr{Q}^{n+1} \quad(n=1,2, \ldots)
$$

This grading of $R$ induces a grading of $\operatorname{Gr}(N)$ of $N$, again in the usual way:

$$
\operatorname{Gr}(N)=\bigoplus_{n=0}^{\infty} \operatorname{Gr}_{n}(N),
$$

where

$$
\mathrm{Gr}_{n}(N)=N \mathscr{Q}^{n} / N \mathscr{Q}^{n+1}
$$

Then $\operatorname{Gr}(N)$ is a graded $\operatorname{Gr}(R)$-module. The Poincaré series of $\operatorname{Gr}_{n}(N)$ is, by definition, the series

$$
r(N, t)=\sum_{n=0}^{\infty} \operatorname{dim}\left(\mathrm{Gr}_{n}(N)\right) t^{n}
$$

Notice that $\operatorname{dim}\left(\mathrm{Gr}_{n-n_{0}}(N)\right)=r(n, G)\left(n \geq n_{0}\right)$. Now $G$ is finitely generated and $G / A=H$ is torsion-free nilpotent. The proof of Theorem 1.9 is now a consequence of the following proposition

PROPOSITION 2. Let $H$ be a finitely generated torsion-free nilpotent group, $R$ the rational group algebra of $H$ over $\mathbb{Q}, \mathscr{Q}$ the augmentation ideal of $R$ and $L=\sum_{n=0}^{\infty} L_{n}$ a finitely generated graded $\mathrm{Gr}(R)$-module. Then the Poincaré series for $L$ is a rational function.

PROOF. The proof of Proposition 2 follows exactly along the same lines as the proof of Theorem 2.2 of [10], with $\mathscr{Q}$ replaced by a field of characteristic $p>0$ and so it will be omitted here (see also $[2,4]$ ). We note only that it follows immediately from the proof of Proposition 2 (see [10] or [2]) that if $G$ is a metabelian $\mathscr{T}$-group, if $A$ is a maximal, Abelian normal subgroup of $G$ containing the derived group of $G$, then

$$
\sigma(t)=f(t) /(1-t)^{h}
$$

where $h$ is at most the rank of the free Abelian group $G / A$ and $f(t)$ is an integral polynomial in $t$. It follows that $s(n)$ is a polynomial in $n$ when $n$ is sufficiently large. These remarks establish (1) and (2) of Corollary 1 (see Section 1.6).

In order to prove (3), notice that, for sufficiently large values of $n, s(n, G)$ is a polynomial in $n$ of degree $d$, say. We need to show that $d=q$. Observe then that it suffices by $[14$, Lemma 1.5 (b)] to prove that for sufficiently large values of $n, r(n, G)$ 
is a polynomial in $n$ of degree $q-1$. Now (see for example $[24$, pages 107,108$]$ ) $\gamma_{n}(G)$ is freely generated, modulo $\gamma_{n+1}(G)$, by the left-normed basic commutators of weight $n$. These commutators take the form

$$
[a_{k}, a_{j}, \underbrace{a_{1}, \ldots, a_{1}}_{i_{1}}, \underbrace{a_{2}, \ldots, a_{2}}_{i_{2}}, \ldots, \underbrace{a_{q}, \ldots, a_{q}}_{i_{q}}]\left(2 \leq k>j, i_{1}+\cdots+i_{q}=n-2\right) .
$$

Consider now the basic commutators of weight $n+1$ of the form

$$
[a_{2}, a_{1}, \underbrace{a_{1}, \ldots, a_{1}}_{i_{1}}, \underbrace{a_{2}, \ldots, a_{2}}_{i_{2}}, \ldots, \underbrace{a_{q}, \ldots, a_{q}}_{i_{q}}]\left(i_{1}+\cdots+i_{q}=n-1\right) .
$$

The number of such commutators is (see [14, Example 1.6])

$$
\left(\begin{array}{c}
n-1+q-1 \\
q-1
\end{array}\right)
$$

a polynomial in $n$ of degree $q-1$. On the other hand, the number of all possible basic commutators is bounded above by

$$
(q-1) q\left(\begin{array}{c}
n-1+q-1 \\
q-1
\end{array}\right)
$$

which is also a polynomial in $n$ of degree $q-1$. So $r(n, G)$ is a polynomial in $n$ of degree $d=q-1$, which completes the proof.

3.3. Finitely generated metanilpotent groups We come next to the proof of Theorem 1.10 .

THEOREM 1.10. Let $G$ be a finitely generated Abelian-by-nilpotent group and let

$$
\Lambda(G)=\bigoplus_{n=1}^{\infty} \gamma_{n}(G) / \gamma_{n+1}(G)
$$

Then the following hold.

(1) The torsion subgroup of $\Lambda(G)$ is of finite exponent.

(2) For each prime $p$, if $e(n, p)$ is the order of the Sylow p-subgroup of the group $\gamma_{n}(G) / \gamma_{n+1}(G)$, then for large values of $n, e(n, p)$ is a polynomial in $n$.

(3) If, in addition, $G$ is polycyclic, then $\gamma_{n}(G) / \gamma_{n+1}(G)$ is ultimately of a fixed finite order.

Proof. Since $G$ is Abelian-by-nilpotent, there exists an integer $n_{0}$ such that $\gamma_{n_{0}}(G)$ is Abelian. Following on much of the notation introduced at the beginning of Section 3.2, we put $A=\gamma_{n_{0}}(G), H=G / A$, denote the integral group algebra of $H$ by 
$R, A$, viewed as an $R$-module, by $M$ and the augmentation ideal of $R$ by $\mathscr{Q}$. Notice that we are here using the integral group algebra of $H$, not the rational group algebra. Finally we view

$$
\operatorname{Gr}(R)\left(=\operatorname{Gr}_{\mathscr{Q}}(R)\right)=\bigoplus_{n=0}^{\infty} \mathscr{Q}^{n} / \mathscr{Q}^{n+1}
$$

as a graded ring and

$$
\operatorname{Gr}(M)=\bigoplus_{n=0}^{\infty} M_{n} / M_{n+1}
$$

where $M_{n}=M \mathscr{Q}^{n}$ as a graded $\operatorname{Gr}(R)$-module. Observe next that for each $n \geq n_{0}$, $\gamma_{n}(G)=M_{n-n_{0}}$.

Now $G$ is finitely generated and $Q$ is a finitely generated nilpotent group. Consequently, $R$ is noetherian (Hall [12]) and $\operatorname{Gr}(R)$ and $\operatorname{Gr}(M)$ are also noetherian (see for example $[2,10])$. The first assertion of Theorem 1.10 follows from this observation.

The proof of the second assertion follows that of Theorem 1.9 (see [10]) and its corollary with only minor changes, using the more general version of the Hilbert-Serre theorem as described in [2].

We are left only with the proof that if $G$ is a polycyclic group, then $\gamma_{n}(G) / \gamma_{n+1}(G)$ is ultimately of a fixed finite order. Now Robinson [26] has observed that $\gamma_{n}(G) / \gamma_{n+1}(G)$ is ultimately of finite order. But we have proved that $\Lambda(G)$ is of finite exponent. It follows that $\gamma_{n}(G) / \gamma_{n+1}(G)$ is of bounded exponent, independent of $n$. Furthermore, the number of generators of $\gamma_{n}(G) / \gamma_{n+1}(G)$ is bounded by the polycyclic length of $G$. This completes the proof of Theorem 1.10.

\section{Lower central dimension and growth}

4.1. Growth functions We give here another proof of the assertion in Theorem 1.10 that every non-negative integer can arise as the lower central dimension of a $\mathscr{T}$-group.

LEMMA 5. Let $X=A$ ? $T$ be the standard wreath product of the infinite cyclic group $A$ by the free Abelian group $T$ of finite rank $q$. Then

(1) $s(n, X)$ is a polynomial in $n$ of degree $q$;

(2) $\delta(X)=q$.

Let $A$ be generated by $a_{q+1}$ and let $a_{1}, \ldots, a_{q}$ be a basis for $T$. We note that $\gamma_{n}(X)$ is, modulo $\gamma_{n+1}(X)$ a free Abelian group with basis those left-normed basic 
commutators of weight $n$ in which $a_{q+1}$ appears at the beginning and occurs only once. It follows that

$$
\bar{\lambda}_{n}(X) \cong \mathscr{Q}^{n} / \mathscr{Q}^{n+1},
$$

where $\mathscr{Q}$ is the augmentation ideal of the integral group ring of $T$. So ([14, Example 1.6])

$$
r(n, X)=\left(\begin{array}{c}
n+q-1 \\
q-1
\end{array}\right) .
$$

This implies that $r(n, X)$ is a polynomial in $n$ of degree $q-1$ and therefore that $s(n, X)$ is a polynomial in $n$ of degree $q$ (see for example [14, page 10]). Hence $\delta(X)=q$.

4.2. Finitely generated metanilpotent groups In this section we concern ourselves with the proof of Theorem 1.11 stated in Section 1.7.

THEOREM 1.11. The lower central dimension of a finitely generated, metanilpotent group is finite.

PROOF. Let $G$ be a finitely generated group, not necessarily in $\mathscr{T}$, containing a nilpotent normal subgroup $L$ such that $G / L$ is nilpotent. Then it suffices for the proof of Theorem 1.11, to show that the torsion-free rank of $G / \gamma_{n}(G)$, that is, $s(n, G)$, is bounded by a polynomial in $n$. In fact we will prove that if the torsion-free rank of $G / L$ is $q$ and if $L$ is nilpotent of class $c$, then the torsion-free rank $s(n)$ of $G / \gamma_{n}(G)$ is bounded by a polynomial of degree $c q$ in $n$.

The proof is by induction on $c$. If $c=1, G$ is a finitely generated Abelianby-nilpotent group. We may assume without loss of generality that $G \in \mathscr{T}$. So, as already noted at the beginning of Section 3.2, we can find an Abelian normal subgroup $A$ of $G$ such that $G / A$ is torsion-free nilpotent. We adopt now the notation of that section and observe, as noted there, that

$$
r(n)=\operatorname{dim}\left(N \mathscr{Q}^{n-n_{0}} / N \mathscr{Q}^{n-n_{0}+1}\right) \quad\left(n \geq n_{0}\right) .
$$

Since $G$ is finitely generated and $G / \gamma_{n_{0}}(G)$ is nilpotent, the Abelian group $\gamma_{n_{0}}(G)$ is a finitely generated $G / A$-module. It follows that the right $R$-module $N$ is also finitely generated, say by $y_{1}, \ldots, y_{k}$. We recall that $\mathscr{Q}$ is the augmentation ideal of the rational group algebra of the torsion-free nilpotent group $G / A$. Hence, by a theorem of Jennings [13], $\mathscr{Q} / \mathscr{Q}^{j}$ is spanned, qua vector space over the field of rational numbers, by all products of the form

$$
\left(x_{1}-1\right)^{i_{1}} \cdots\left(x_{q}-1\right)^{i_{q}} \quad\left(i_{1}+\cdots+i_{q}=j-1\right),
$$


where here

$$
1<g p\left(x_{q} A\right)<g p\left(x_{q} A, x_{q-1} A\right)<g p\left(x_{q} A, x_{q-1} A, \ldots, x_{1} A\right)=G / A
$$

is a central series for $G / A$ with infinite cyclic factors. The number of such products is bounded by

$$
\left(\begin{array}{c}
j+q-1 \\
q-1
\end{array}\right)
$$

a polynomial of degree $q-1$ in $j$ (see the proof of Lemma 5). Since $N$ is generated, qua $R$-module, by $y_{1}, \ldots, y_{k}$ it follows that the dimension over $\mathbb{Q}$ of

$$
N \mathscr{Q}^{n-n_{0}} / N \mathscr{Q}^{n-n_{0}+1} \quad\left(n>n_{0}\right)
$$

is at most

$$
k \times \operatorname{dim}\left(\mathscr{Q}^{n-n_{0}} / N \mathscr{Q}^{n-n_{0}+1}\right)
$$

Consequently $r(n)$ is bounded by a polynomial in $n$ of degree $q-1$. Therefore (see the proof of Lemma 5) $s(n)$ is bounded by a polynomial of degree $q$ in $n$.

Inductively we can assume that the torsion-free rank of

$$
G / \gamma_{c}(L) / \gamma_{n}\left(G / \gamma_{c}(L)\right)
$$

is bounded by a polynomial in $n$ of degree $(c-1) q$. Now

$$
\gamma_{n}\left(G / \gamma_{c}(L)\right)=\gamma_{n}(G) \gamma_{c}(L) / \gamma_{c}(L)
$$

Hence

$$
G / \gamma_{c}(L) / \gamma_{n}\left(G / \gamma_{c}(L)\right) \cong G / \gamma_{n}(G) \gamma_{c}(L) \cong G / \gamma_{n}(G) / \gamma_{c}\left(L \gamma_{n}(G) / \gamma_{n}(G)\right)
$$

So if we put $G_{n}=G / \gamma_{n}(G)$ and $L_{n}=L \gamma_{n}(G) / \gamma_{n}(G)$, then the inductive assumption amounts to the observation that the torsion-free rank of $G_{n} / \gamma_{c}\left(L_{n}\right)$ is bounded by a polynomial in $n$ of degree $(c-1) q$. It follows that the torsion-free rank of $\gamma_{c-1}\left(L_{n}\right)$ is bounded, modulo $\gamma_{c}\left(L_{n}\right)$, by a polynomial in $n$ of degree $(c-1) q$. This implies that we can find, for each $n$, a set $X_{n}$ of $\mathrm{O}\left(n^{(c-1) q}\right)$ elements of $\gamma_{c-1}\left(L_{n}\right)$ which generates, modulo $\gamma_{c}\left(L_{n}\right)$, a subgroup of finite index. Our initial observation about Abelianby-nilpotent groups translates, with our current notation, into the assertion that the torsion-free rank of $G_{n} /\left[L_{n}, L_{n}\right]$ is bounded by a polynomial in $n$ of degree $q$. Hence the torsion-free rank of $L_{n} /\left[L_{n}, L_{n}\right]$ is also bounded by a polynomial in $n$ of degree 
q. This implies that we can find, for each $\mathrm{n}$, a set $Y_{n}$ of $\mathrm{O}\left(n^{q}\right)$ elements of $L_{n}$ which generates, modulo $\left[L_{n}, L_{n}\right.$ ], a subgroup of finite index in $L_{n}$. Consequently

$$
Z_{n}=\left\{[x, y] \mid x \in X_{n}, y \in Y_{n}\right\}
$$

generates, for each $n$ sufficiently large, a subgroup of finite index in $\gamma_{c}\left(L_{n}\right)$. Now the number of elements in $Z_{n}$ is $\mathrm{O}\left(n^{(c-1) q} \cdot n^{q}\right)=\mathrm{O}\left(n^{c q}\right)$. So the torsion-free rank of $G_{n}$ is bounded by a polynomial of degree $n^{c q}$, for all sufficiently large values of $n$, as desired.

\section{The proof of Theorem 1.14}

5.1. Preliminaries The objective of this section is to prepare the ground for the proof of Theorem 1.14.

THEOREM 1.12. Let $G$ be a $\mathscr{T}$-group whose center is not finitely generated and let $\mathscr{T}^{*}$ be the class of all quotient groups of $G$, with infinitely generated centers, which lie in $\mathscr{T}$. Then the set of lower central Poincaré series of groups in $\mathscr{T}^{*}$ has the cardinality of the real line.

We begin with the proof of the following well-known lemma.

LEMMA 6. Let $G \in \mathscr{T}$. Then $G$ is a subdirect product of the finitely generated torsion-free nilpotent groups

$$
G_{n}=G / \bar{\gamma}_{n}(G)
$$

Let $P=\prod_{n} G_{n}$ be the unrestricted direct product of the factor groups $G_{n}$. Then the mapping $\mu$ which sends $g \in G$ to that element of $P$ whose $n$th coordinate is $g \bar{\gamma}_{n}(X)$ is the required injection of $G$ into $P$. We adopt until further notice the notation used above.

The next lemma will be of use in the sequel.

LEMMA 7. The center $C$ of $G$ is free Abelian.

PROOF. $C$ is a subgroup of the unrestricted direct product of the centers $C_{n}$ of the groups $G_{n}$. Now each $C_{n}$ is free Abelian of finite rank. Therefore $C$ can be embedded in the unrestricted direct product of a countable number of copies of the infinite cyclic group. Since $C$ is countable, it follows from a theorem of Specker [28] that $C$ is free Abelian.

This brings me to the following important observation. 
LEMMA 8. Let $H$ be a subgroup of $C$, the center of $G$ and let $H_{n}$ be the image of $H$ under the canonical homomorphism of $G$ onto $G_{n}$. Let $I_{n} / \bar{\gamma}_{n}(G)$ be the isolator of $H_{n}$ in $G / \bar{\gamma}_{n}(G)$. Then

$$
\bar{\gamma}_{n}(G / H)=I_{n} / H
$$

and hence

$$
(G / H) /\left(\bar{\gamma}_{n}(G / H)\right) \cong G / I_{n}
$$

PROOF. $\gamma_{n}(G / H)=\gamma_{n}(G) H / H$. So the torsion subgroup of $(G / H) /\left(\gamma_{n}(G / H)\right)$ contains $\bar{\gamma}_{n}(G) H / H$ and therefore also any element of finite order modulo $\bar{\gamma}_{n}(G) H / H$. This means that the torsion subgroup of $(G / H) /\left(\gamma_{n}(G / H)\right)$ contains $I_{n} / \bar{\gamma}_{n}(G) H$, that is, by definition $\bar{\gamma}_{n}(G / H) \geq I_{n} / H$. On the other hand,

$$
G / H / I_{n} / H \cong G / I_{n}
$$

is a torsion-free nilpotent group of class at most $n-1$. Hence, $I_{n} / H \geq \bar{\gamma}_{n}(G / H)$, which implies that

$$
I_{n} / H=\bar{\gamma}_{n}(G / H)
$$

This completes the proof of the lemma.

We shall need the following consequence of Lemma 8. We again adopt the notation introduced previously.

LEMMA 9. Suppose that $H=g p\left(a_{1}, a_{2}, \ldots\right)$ and that there are integers $\alpha$ and $\beta$ such that $a_{j} \in \bar{\gamma}_{\alpha}(G)$ whenever $j>\beta$. Let $h_{n}$ be the torsion-free rank of $G / \bar{\gamma}_{n}(G)$ and let $h_{n}^{\prime}$ be the torsion-free rank of $(G / H) /\left(\bar{\gamma}_{n}(G / H)\right)$. Then

$$
h_{n}-h_{n}^{\prime} \leq \beta \text { for every } n \leq \alpha .
$$

PROOF. It follows immediately from the hypothesis that if $n \leq \alpha$ then $H_{n}$ is generated by the images of $a_{1}, a_{2}, \ldots, a_{\beta}$ in $G_{n}$. So $H_{n}$ is then a free Abelian group of rank at most $\beta$. Consequently its isolator, $I_{n}$ modulo $\bar{\gamma}_{n}(G)$ is free Abelian of rank at most $\beta$. By Lemma $8, I_{n}=\bar{\gamma}_{n}(G) H$. This completes the proof of the lemma.

Next we prove the following lemma.

LEMMA 10. Let $C_{n}$ be the image of $C$ under the canonical homomorphism of $G$ onto $G_{n}$ and let $\rho_{n}$ be the rank of the free Abelian group $C_{n}$. Then given any positive integer $n$ we can find an integer $\alpha_{n}$ such

$$
\rho_{m} \geq n \text { whenever } m \geq \alpha_{n} \text {. }
$$


PROOF. It suffices to prove that the sequence

$$
\rho_{1}, \rho_{2}, \ldots
$$

does not become constant. Let then $n$ be any given positive integer. Now $C$ maps onto $C_{n}$ under the canonical homomorphism $\mu_{n}$ of $G$ onto $G_{n}$. This gives rise to a splitting of $C$ into a direct product

$$
C=D \times E
$$

where $D$ is the kernel of $\mu_{n}$. Since $E \cong C_{n}$ is finitely generated and $G \in \mathscr{T}^{*}, D \neq 1$. Let $d \in D, d \neq 1$. There exists an integer $m>n$ such that $d \notin \bar{\gamma}_{m}(G)$. This means that there is a homomorphism of $C_{m}$ onto $C_{n}$ with a non-trivial kernel. So $\rho_{m}>\rho_{n}$, as desired.

We end this section by proving two results involving the centers of $\mathscr{T}$-groups. The first of these is probably well-known.

LEMMA 11. Suppose that the group $G$ is residually torsion-free nilpotent. Then $G / C$ is also residually torsion-free nilpotent, where here $C$ is the center of the group G.

PROOF. $G$ acts by conjugation on itself. This induces a faithful representation of $G / C$ on $G$ which stabilises the series

$$
G \geq \bar{\gamma}_{2}(G) \geq \bar{\gamma}_{3}(G) \geq \cdots
$$

So $G / C$ is residually torsion-free nilpotent.

This brings me to the next lemma.

LEMMA 12. Let $G \in \mathscr{T}$ and let $H$ be an isolated cyclic subgroup of the center of G. Then $G / H \in \mathscr{T}$.

Proof. Let $H_{n}$ be the image of $H$ under the canonical homomorphism of $G$ onto $G_{n}$ and let $I_{n}$ be its isolator in $G_{n}$. Then $I_{n}$ is a cyclic subgroup in the center of $G_{n}$ and $G_{n} / I_{n}$ is a torsion-free nilpotent group. Let $I$ be the unrestricted direct product of the groups $I_{n}$, let $P$ be the unrestricted direct product of the groups $G_{n}$ and let $\mu$ be the injection of $G$ into $P$ defined in the course of the proof of Lemma 6 . We can view $I$ as a subgroup of $P$. Then $I$ is in the center of $P$ and $P / I$ is the unrestricted direct product of the groups $G_{n} / I_{n}$ :

$$
P / I=\prod_{n} G_{n} / I_{n}
$$


Consider now $J=\mu^{-1}(I \cap \mu(G))$. $J$ is a subgroup of the center of $G$ and $J$ contains $H$. We claim that $J=H$. To see this, notice first that $J$ is an isolated subgroup of $G$ because $I \cap \mu(G)$ is isolated in $P$. Now if $J$ is cyclic, then $J=H$ because $H$ is a maximal cyclic subgroup of $G$. Suppose, if possible, that $J$ is not cyclic. Then $J$ is free Abelian, since it is a subgroup of the free Abelian group $C$. Since $H$ is an isolated subgroup of $J, H$ is a direct factor of $J$. So we can find a subgroup $L$ of $J$ containing $H$ which is free Abelian of rank two. Notice that $L$ projects onto the cyclic group $I_{n}$, for every $n$. If we choose $n$ sufficiently large, say $n \geq n_{0}$, then $I_{n}$ is non-trivial. Now $I_{n_{0}}$ is infinite cyclic. So we can find a basis $\{a, b\}$ for $L$ so that the canonical image of $a$ generates $I_{n_{0}}$ and $b \in \bar{\gamma}_{n_{0}+1}(G)$. Since $G \in \mathscr{T}$, we can find an integer, say $m>n_{0}+1$, so that $b \notin \bar{\gamma}_{m}(G)$. But then $a$ and $b$ generate modulo $\bar{\gamma}_{m}(G)$ a free Abelian group of rank two. On the other hand they generate, modulo $\bar{\gamma}_{m}(G)$, a subgroup of the cyclic group $I_{m}$, which is a contradiction. This implies then that $J=H$. Consequently the canonical homomorphism of $G$ into $P / I$ has kernel $H$. So we have proved that $G / H \in \mathscr{T}$.

This then yields the following theorem

THEOREM 5.1. Let $G \in \mathscr{T}$ and let $H$ be a finitely generated isolated subgroup of the center of $G$. Then $G / H \in \mathscr{T}$.

It is perhaps worth noting that it is not possible to relax the condition on $H$ that it be finitely generated. Indeed suppose that $G \in \mathscr{T}^{*}$. Then the center $C$ of $G$ maps onto the additive group of rational numbers with kernel $K$, say. $K$ is then an isolated subgroup of the center of $G$, but $G / K \notin \mathscr{T}$ because, by Lemma 7, the center of a group in $\mathscr{T}$ is always free Abelian.

5.2. The proof of Theorem 1.14 Suppose, if possible, that the set of lower central Poincaré series of $\mathscr{T}^{*}$-groups is countable. We can then enumerate a sequence of groups

$$
G(1), G(2), \ldots
$$

in $\mathscr{T}^{*}$ whose lower central Poincare series are in one-to-one correspondence with this set. We will construct a new group $X \in \mathscr{T}^{*}$ whose lower central Poincaré series is different from every one of the $G(i)$. Our group $X$ is obtained from $G$ by factoring out an appropriate subgroup $Y$ of $G$. The subgroup $Y$ is obtained by first concocting the union $H$ of a sequence $H_{1}, H_{2}, \ldots$ of subgroups $H_{i}$ of the center $C$ of $G$ in a suitable way; $Y$ then is the preimage in $G$ of $\bar{\gamma}_{\omega}(G / H)$.

We begin by considering the group $G(1)$. If the lower central Poincaré series of $G$ coincides with that of $G(1)$ from some point on, we proceed as follows. We choose, by Lemma 10 , an integer $n_{1}>1$ so that the image $C_{n_{1}}$ of $C$ in $G_{n_{1}}$ has rank at 
least two and so that the $n_{1}$-th coefficient of the lower central Poincaré series of $G(1)$ coincides with the $n_{1}$-th coefficient of the lower central Poincaré series of $G$. Now choose an element $a_{1} \in \bar{\gamma}_{n_{1}}(G) \cap C, a_{1} \notin \bar{\gamma}_{n_{1}+1}(G)$ so that $H(1)=g p\left(a_{1}\right)$ is an isolated subgroup of $G$. Observe that, by Lemma $12, G / H(1) \in \mathscr{T}$, that the rank of the canonical image of $C_{n_{1}}$ in $G(1)$ is at least one and that the $n_{1}$-th coefficient of the lower central Poincaré series of $G / H(1)$ is one less than the $n_{1}$-th coefficient of the lower central Poincaré series of $G(1)$. Hence the lower central Poincaré series of $G(1)$ and that of $G / H(1)$ have different $n_{1}$-th coefficients. If infinitely many of the coefficients of the lower central Poincaré series of $G$ differ from the corresponding coefficients of the lower central Poincaré series of $G(1)$, put $a_{1}=1$ and $H_{1}=1$ and choose $n_{1}>1$ large enough so that the rank of $C_{n_{1}}$ is at least one and the lower central Poincaré series of $G(1)$ and $G / H(1)$ have different $n_{1}$-th coefficients.

Now suppose that we have chosen a sequence

$$
n_{1}<n_{2}<\cdots<n_{m}
$$

of integers and a corresponding sequence

$$
a_{1}, a_{2}, \ldots, a_{m}
$$

of elements of $G$ in such a way that either $a_{j}=1$ or else

$$
a_{j} \in \bar{\gamma}_{n_{j}}(G) \cap C, \quad a_{j} \notin \bar{\gamma}_{n_{1}+1}(G)
$$

for $j=1,2, \ldots, m$, that

$$
H(j)=g p\left(a_{1}, a_{2}, \ldots, a_{j}\right)
$$

is an isolated subgroup of $G$ for each $j=1, \ldots, m$, that the rank of the canonical image of $C_{n_{j}}$ in $G / H(j)$ is at least $j$ and so that the lower central Poincaré series of $G / H(j)$ differs from that of $G(j)$ in the $n_{j}$-th coefficient for $j=1, \ldots, m$. We need now to choose $n_{m+1}$ and $a_{m+1}$. If the lower central Poincaré series of $G / H(m)$ ultimately coincides with the lower central Poincaré series of $G(m+1)$, choose $n_{m+1}>n_{m}$ large enough to ensure that the rank of $C_{n_{m+1}}$ is at least $2(m+1)$ and so that we can find

$$
a_{m+1} \in \bar{\gamma}_{n_{m+1}}(G) \cap C, \quad a_{m+1} \notin \bar{\gamma}_{n_{m+1}+1}(G)
$$

with $H(m+1)=g p\left(a_{1}, \ldots, a_{m+1}\right)$ an isolated subgroup of $G$. Then the lower central Poincaré series of $G / H(m+1)$ has the same coefficients as $G / H(m)$ up to and including the $\left(n_{m+1}-1\right)$-th coefficient and the $n_{m+1}$-th coefficient of $G / H(m+1)$ is one less than that of the $n_{m+1}$-th coefficient of $G(m+1)$. Moreover the rank of 
the canonical image of $C_{n_{m+1}}$ in $G / H(m+1)$ is at least $m+1$. If the lower central Poincaré series of $G / H(m)$ differs from that of $G(m+1)$ in infinitely many places, then we choose $a_{m+1}=1, n_{m+1}>n_{m}$ large enough to ensure that the rank of $C_{n_{m+1}}$ is at least $m+1$ and such that the lower central Poincaré series of $G(m+1)$ and $G / H(m)$ have different $n_{m+1}$-th coefficients. This then completes the definition of the sequence $n_{1}, n_{2}, \ldots$ and the elements $a_{1}, a_{2}, \ldots$

We now define $H$ to be the union of the $H(i)$ and define $Y$ by

$$
\bar{\gamma}_{\omega}(G / H)=Y / H \text {. }
$$

Then the lower central Poincaré series of $G / Y$ differs from that of each $G(m)$ as we have arranged matters in such a way as to ensure that the $n_{m}$-th coefficient of the lower central Poincaré series of $G / Y$ is different from the $n_{m}$-th coefficient of the Poincaré series of $G(m)$. Finally we need only to observe that the canonical projection of $C$ into $G / Y$ is not finitely generated. Indeed $C$ maps onto a subgroup of $(G / Y) / \bar{\gamma}_{n_{m}}(G / Y)$ which is free Abelian of rank at least $m$ for every $m$. So $G / Y \in \mathscr{T}^{*}$ and this then completes the proof of Theorem 1.14.

5.3. A group of Philip Hall Let $\mathbf{P}=\mathbb{Q}[[y]]$ be the algebra of power series in $y$ with coefficients in the field $\mathbb{Q}$ of rational numbers and let $M=M(3, \mathbf{P})$ be the $\mathbb{Q}$-algebra of all $3 \times 3$ matrices over P. Consider the elements

$$
\tau=\left(\begin{array}{lll}
0 & 0 & 0 \\
0 & y & 0 \\
0 & 0 & 0
\end{array}\right), \quad \alpha=\left(\begin{array}{lll}
0 & 0 & 0 \\
1 & 0 & 0 \\
1 & 1 & 0
\end{array}\right)
$$

of $M$. Observe that $t=1+\tau$ and $a=1+\alpha$ are invertible elements of $M$. We put

$$
G=g p(t, a) .
$$

$G$ is a group that goes back to Hall [12]. Our claim here is that $G \in \mathscr{T}^{*}$. In order to see how this comes about, notice that

$$
\alpha^{3}=\tau \alpha^{2}=\alpha^{2} \tau=\tau \alpha \tau=0 .
$$

Let $\Lambda_{0}$ be the $\mathbb{Q}$-subspace of $M$ spanned by the identity matrix and let $\Lambda_{n}$ be the $\mathscr{Q}$-subspace of $M$ spanned by the matrices

$$
\tau^{n}, \alpha \tau^{n-1}, \tau^{n-1} \alpha, \alpha \tau^{n-2} \alpha .
$$

It follows from (1) that the elements in (2) form a basis for $\Lambda_{n}$. Let $\mathbf{P}_{n}$ be the ideal of $\mathbf{P}$ consisting of all those power series $c_{0}+c_{1} y+\cdots+c_{j} y^{j}+\cdots$ for which $c_{0}=c_{1}=\cdots=c_{n}=0$. Consider the canonical homomorphism of $M(3, \mathbf{P})$ into 
$M\left(3, \mathbf{P} / \mathbf{P}_{n}\right)$. The images of $\tau$ and $\alpha$ in $M\left(3, \mathbf{P} / \mathbf{P}_{n}\right)$ generate a nilpotent $\mathbb{Q}$-subalgebra and hence the image of $G$ in $M\left(3, \mathbf{P} / \mathbf{P}_{n}\right)$ is a torsion-free nilpotent group. Since the $\mathbf{P}_{n}$ intersect in 0 , it follows that $G \in \mathscr{T}$. We leave it to the reader to verify that the center of $G$ is free Abelian of infinite rank, freely generated by the commutators $\left[a, t^{-n} a t^{n}\right](n \in \mathbb{Z})$ and hence that $G \in \mathscr{T}^{*}$.

5.4. A final comment The point of our main theorem, Theorem 1.14 , is that there are a host of groups with different lower central Poincaré series. The question arises as to whether groups with the same lower central Poincare series have anything in common. In particular it is still unknown whether a group in $\mathscr{T}$ with the same lower central Poincaré series as a free group is finitely related. We cite here the paper [5] for some interesting new examples of this kind.

\section{References}

[1] R. C. Alperin and B. L. Peterson, 'Growth functions for residually torsion-free nilpotent groups', Proc. Amer. Math. Soc. 109 (1990), 585-587.

[2] M.F. Atiyah and I. G. Macdonald, Introduction to commutative algebra (Addison-Wesley, Reading, 1969).

[3] G. Baumslag, 'On generalized free products', Math. Z. 78 (1962), 423-438.

[4] _ - 'Reflections on metabelian groups', Contemp. Math. 109 (1990), 1-9.

[5] _ _ 'Musings on Magnus', Contemp. Math. 169 (1994), 99-106.

[6] __..., 'Finitely generated residually torsion-free nilpotent groups. II', Technical Report.

[7] N. Blackburn, 'Conjugacy in nilpotent groups', Proc. Amer. Math. Soc. 16 (1965), 143-148.

[8] P. M. Cohn, Free rings and their relations (Academic Press, London, 1971).

[9] M. Gromov, 'Hyperbolic groups', in: Essays on group theory (ed. S. M. Gersten), Math. Sci. Res. Inst. Publ. 8 (Springer, New York, 1987).

[10] J. R. J. Groves and J. S. Wilson, 'Finitely presented metanilpotent groups', J. London Math. Soc. 50 (1994), 87-104.

[11] K. W. Gruenberg, 'Residual properties of infinite soluble groups', Proc. London Math. Soc. (3) 7 (1957), 29-62.

[12] P. Hall, 'Finiteness conditions for soluble groups', Proc. London Math. Soc. (3) 4 (1954), 419-436.

[13] S. A. Jennings, 'The group ring of a class of infinite nilpotent groups', Canad. J. Math. 7 (1955), 169-187.

[14] G. R. Krause and T. H. Lenagan, Growth of algebras and Gelfand-Kirillov dimension, Research Notes in Math. 166 (Pitman, Berlin, 1985).

[15] A. G. Kurosh, The theory of groups, I, II, 2nd edition, translated and edited by K. A. Hirsch (Chelsea, New York, 1960).

[16] A. I. Lichtman, 'The residual nilpotence of the multiplicative group of a skew field generated by universal enveloping algebras', J. Algebra 112 (1988), 250-263.

[17] W. Magnus, 'Beziehungen zwischen Gruppen und Idealen in einem speziellen Ring', Math. Ann. 111 (1935), 259-280.

[18] W. Magnus, A. Karrass and D. Solitar, Combinatorial group theory (Wiley, New York, 1966).

[19] A. I. Malčev, 'Generalized nilpotent algebras and their associated groups', Mat. Sbornik (N.S.) 25 (67) (1949), 347-366. 
[20] C. F. Miller, On group-theoretic decision problems and their classification, Ann. of Math. Studies 68 (Princeton University Press, Princeton, 1971).

[21] _ - 'Decision problems for groups-survey and reflections', in: Algorithms and classification in combinatorial group theory (eds. G. Baumslag and C. F. Miller III), Math. Sci. Res. Inst. Publ. 23 (Springer, Berlin, 1990).

[22] J. Milnor, 'A note on curvature and fundamental groups', J. Differential Geom. 2 (1968), 1-7.

[23] B. H. Neumann, H. Neumann and P. Neumann, 'Wreath products and varieties of groups', Math. Z. 80 (1962), 44-62.

[24] H. Neumann, Varieties of groups, Ergebnisse der Mathematik, Bd. 37 (Springer, New York, 1967).

[25] M. O. Rabin, 'Recursive unsolvability of group theoretic problems', Ann. of Math. (2) 67 (1958), 172-194.

[26] D. J. S. Robinson, A course in the theory of groups, Graduate Texts in Math. 80 (Springer, New York, 1982).

[27] D. Segal, Polycyclic groups (Cambridge University Press, Cambridge, 1983).

[28] E. Specker, 'Additive Gruppen von Folgen ganzer Zahlen', Portugal. Math. 9 (1950), 131-140.

\author{
Department of Mathematics \\ City College of New York \\ New York, NY 10031 \\ USA
}

